\title{
KL-6 mucin is a useful immunohistochemical marker for cholangiocarcinoma
}

\author{
WEI TANG ${ }^{1,2}$, QIAN GUO $^{1}$, XIANJUN QU ${ }^{1,2}$, YOSHINORI INAGAKI ${ }^{3}$, \\ YASUJI SEYAMA ${ }^{1}$, YUTAKA MIDORIKAWA ${ }^{1}$, RUOYAN GAI ${ }^{1}$, NORIHIRO KOKUDO ${ }^{1}$, \\ YASUHIKO SUGAWARA ${ }^{1}$, MUNEHIRO NAKATA ${ }^{3}$ and MASATOSHI MAKUUCHI ${ }^{1}$ \\ ${ }^{1}$ Hepato-Biliary-Pancreatic Surgery Division, Department of Surgery, Graduate School of Medicine, \\ the University of Tokyo, Tokyo, Japan; ${ }^{2}$ Department of Pharmacology, School of Pharmaceutical Sciences, \\ Shandong University, Jinan, P.R. China; ${ }^{3}$ Department of Applied Biochemistry, Tokai University, Kanagawa, Japan
}

Received August 11, 2006; Accepted September 27, 2006

\begin{abstract}
This study aimed to clarify the clinical significance of the expression of KL-6 mucin, a type of MUC1, in primary liver cancer. Tissue specimens were collected from 21 patients with cholangiocarcinoma (CC), 78 with hepatocellular carcinoma (HCC), and 12 with combined hepatocellular and cholangiocarcinoma (cHCC-CC). Immunohistochemical analysis was done using a monoclonal antibody for KL-6 mucin as well as antibodies for Hep1 or CK7. KL-6 staining was positive in all the $\mathrm{CC}$ tissues examined, while it was not positive in any of the HCC tissues. Similar selectivity of KL-6 staining was also observed in the cHCC-CC specimens, and the cholangiocellular tissue could be clearly delineated by KL-6 staining. In contrast, $79.5 \%$ of HCC specimens and $25.0 \%$ of cHCC-CC specimens were positive for Hep1 in the hepatocellular tissues, while none of the CC or cHCC-CC specimens were positive in the cholangiocellular tissues. Staining for CK7 was positive in $95.2 \%$ of $\mathrm{CC}$ and $35.9 \%$ of HCC specimens, while 58.3 and $25.0 \%$ of cHCC-CC specimens displayed positivity for CK7 in the cholangiocellular and hepatocellular tissues, respectively. These results suggest that KL-6 may be a useful tumor marker for distinguishing CC from HCC. In addition, the high selectivity of KL-6 for cholangiocallular tissue may help to provide information for deciding the clinical strategy in cHCC-CC patients.
\end{abstract}

\section{Introduction}

Primary liver cancer is classified as hepatocellular carcinoma (HCC) or intrahepatic cholangiocarcinoma (CC). Since these

Correspondence to: Dr Wei Tang, Hepato-Biliary-Pancreatic Surgery Division, Department of Surgery, Graduate School of Medicine, the University of Tokyo, 7-3-1 Hongo, Bunkyo-ku, Tokyo 113-8655, Japan

E-mail: tang-sur@h.u-tokyo.ac.jp

Key words: KL-6 mucin, cholangiocarcinoma, hepatocellular carcinoma, liver cancer, immunohistochemistry two cancers differ with respect to their etiological, epidemiological, and clinical characteristics, distinguishing between them is important (1). In addition, various combinations of hepatocellular and cholangiocellular components have been reported in combined hepatocellular and cholangiocarcinoma (cHCC-CC), a rare type of liver cancer (1-3). Immunohistochemical detection of various immunoreactive targets (such as Hep1, CK7, carcinoembryonic antigen, $\alpha 1$-antitrypsin, and fibrinogen), which are expressed by or accumulate in tumor tissues, is often used to assist in making a histopathological diagnosis $(3,4)$. However, the sensitivity and specificity of such methods are still limited when attempting to distinguish HCC from CC.

KL-6 mucin is a type of MUC1, which is recognized by the KL-6 monoclonal antibody (mAb) that was obtained from a hybridoma established from splenocytes of a BALB/c mouse immunized with human pulmonary adenocarcinoma $(5,6)$. Characteristically, sialylation of the carbohydrate moiety on the mucin molecule is essential for recognition by KL-6 mAb (6). KL-6 mucin has not only been detected in adenocarcinoma of the lung but also in various cancer cell lines, secretory epithelial tissues lining the respiratory, reproductive, gastrointestinal tracts, and bile duct, and carcinoma tissues $(6,7)$. We have reported that aberrant expression of KL-6 mucin by primary colorectal carcinoma is correlated with invasion and metastasis of the tumor. We have also found that KL-6 expression can be detected in metastatic liver tissues, but not in the surrounding normal liver or HCC tissues (8). This distinct difference between metastatic liver cancer and HCC suggested that KL- 6 mAb could be used the for discrimination between HCC and CC. We found that KL-6 $\mathrm{mAb}$ stained $\mathrm{CC}$ tissues but not HCC tissues, even in $\mathrm{cHCC}-$ $\mathrm{CC}$ lesions, suggesting that immunohistochemical detection of KL-6 mucin is useful for distinguishing between these two types of tumors.

\section{Materials and methods}

Patients. Liver tissue samples were collected from $21 \mathrm{CC}$ patients (12 men and 9 women with a median age of 58 years and a range of 33-80 years whose tumors ranged from 0.5 to 
Table I. Positive staining for KL-6 mucin, CK7, and Hep1 in CC and HCC.

\begin{tabular}{|c|c|c|c|c|}
\hline \multirow[t]{2}{*}{ Antigen } & \multicolumn{2}{|c|}{$\begin{array}{l}\text { Positive staining of } \mathrm{CC} \\
\qquad(\mathrm{n}=21)\end{array}$} & \multicolumn{2}{|c|}{$\begin{array}{l}\text { Positive staining of } \mathrm{HCC} \\
\qquad(\mathrm{n}=78)\end{array}$} \\
\hline & Cancer tissue & $\begin{array}{l}\text { Surrounding } \\
\text { normal tissue }\end{array}$ & Cancer tissue & $\begin{array}{l}\text { Surrounding } \\
\text { normal tissue }\end{array}$ \\
\hline KL-6 mucin & $21(100 \%)$ & $0(0 \%)$ & $0(0 \%)$ & $0(0 \%)$ \\
\hline CK7 & $20(95.2 \%)$ & $12(57.1 \%)$ & $28(35.9 \%)$ & $51(65.4 \%)$ \\
\hline Hep1 & $0(0 \%)$ & $21(100 \%)$ & $62(79.5 \%)$ & $78(100 \%)$ \\
\hline
\end{tabular}

Table II. Positive staining for KL-6 mucin, CK7, and Hep1 in cHCC-CC ( $\mathrm{n}=12)$.

\begin{tabular}{lccc}
\hline Antigen & \multicolumn{3}{c}{ Positive staining } \\
\cline { 2 - 4 } & Cholangiocellular area & Hepatocellular area & Surrounding normal tissue \\
\hline KL-6 mucin & $12(100 \%)$ & $0(0 \%)$ & $0(0 \%)$ \\
CK7 & $7(58.3 \%)$ & $3(25.0 \%)$ & $8(66.7 \%)$ \\
Hep1 & $0(0 \%)$ & $8(66.7 \%)$ & $12(100 \%)$ \\
\hline
\end{tabular}

$10.0 \mathrm{~cm}$ ), $78 \mathrm{HCC}$ patients (63 men and 15 women with a median age of 61 years and a range of 24-81 years whose tumors ranged from 1.2 to $17.0 \mathrm{~cm}$ ), and $12 \mathrm{cHCC}-\mathrm{CC}$ patients (10 men and 2 women with a median age of 53 years and a range of 21-71 years whose tumors ranged from 3.0 to $9.0 \mathrm{~cm}$ ). Patients underwent surgical resection at the Hepato-BiliaryPancreatic Surgery Division of the Department of Surgery, Graduate School of Medicine, the University of Tokyo between January 1994 and December 2002. Their clinicopathological characteristics were evaluated according to the General Rules for the Clinical and Pathological Study of Primary Liver Cancer (9). The TNM system of the International Union Against Cancer was used for staging (10).

Immunohistochemical staining. Sections (4- $\mu$ m thick) were cut from archival formalin-fixed paraffin-embedded tissue blocks, deparaffinized, and dehydrated using a graded ethanol series. Endogenous peroxidase activity was blocked by incubation with $0.3 \%$ hydrogen peroxide/methanol for $30 \mathrm{~min}$, after which the slides were rinsed with phosphate-buffered saline and then blocked with normal goat serum at room temperature for $30 \mathrm{~min}$. Next, the sections were incubated with anti-KL-6 mucin mIgG (1:200; Eisai, Tokyo, Japan), anti-cytokeratin 7 (CK7) mIgG (1:150; Chemicon, Inc., CA, USA), or antihuman hepatocyte (Hep1) mIgG (1:25; DakoCytomation, Glostrup, Denmark) for $60 \mathrm{~min}$ at room temperature. For Hep1 staining, the slides were pretreated with heat-induced epitope retrieval solution in an autoclave for 20 min before incubation with the antibody. After the sections were incubated with the biotinylated secondary antibody for $30 \mathrm{~min}$, bound antibody was labelled by the biotin-streptavidin-peroxidase complex method using a commercial kit according to the manufacturer's instructions (Histofine SAB-PO kit; Nichirei, Tokyo, Japan).
3,3'-Diaminobenzidine was used as the chromogen, and hematoxylin was employed for counter-staining. Negative control sections were treated by omitting the primary antibody to monitor background staining.

Overall immunohistochemical staining was evaluated for tumor cells in the entire region examined. Subcellular staining patterns were recorded by assessing the extent of staining of the apical membrane, circumferential membrane, and cytoplasm, as described elsewhere (8). Three investigators (W.T., Q.G., and N.K.) separately judged the staining, and discrepancies were resolved through discussion of the findings.

\section{Results}

Immunostaining of CC and HCC tissues for KL-6 mucin. Typical staining profiles of $\mathrm{CC}$ and $\mathrm{HCC}$ tissues that were obtained with KL-6 mAb are shown in Fig. 1A and B, respectively. The number of tumors with positive staining is summarized in Table I. Staining for KL-6 mucin was positive in all CC tissues examined (Table I and right side of Fig. 1A). Staining was observed in the cytosol and cell membrane of CC cells and in a mucinous substance on the luminal surface of the glandular structures (Fig. 2). In contrast, staining for KL-6 mucin was not observed in HCC tissues (right side of Fig. 1B) or in the surrounding normal hepatic parenchyma (left side of Fig. 1A and B), although some positive staining was observed in the mucinous substance found in normal bile ducts and on the apical surface of non-cancerous cholangiocytes (data not shown).

Immunostaining of CC and HCC tissues for CK7 and Hepl. Staining for CK7 was found in some CC and HCC tissues 

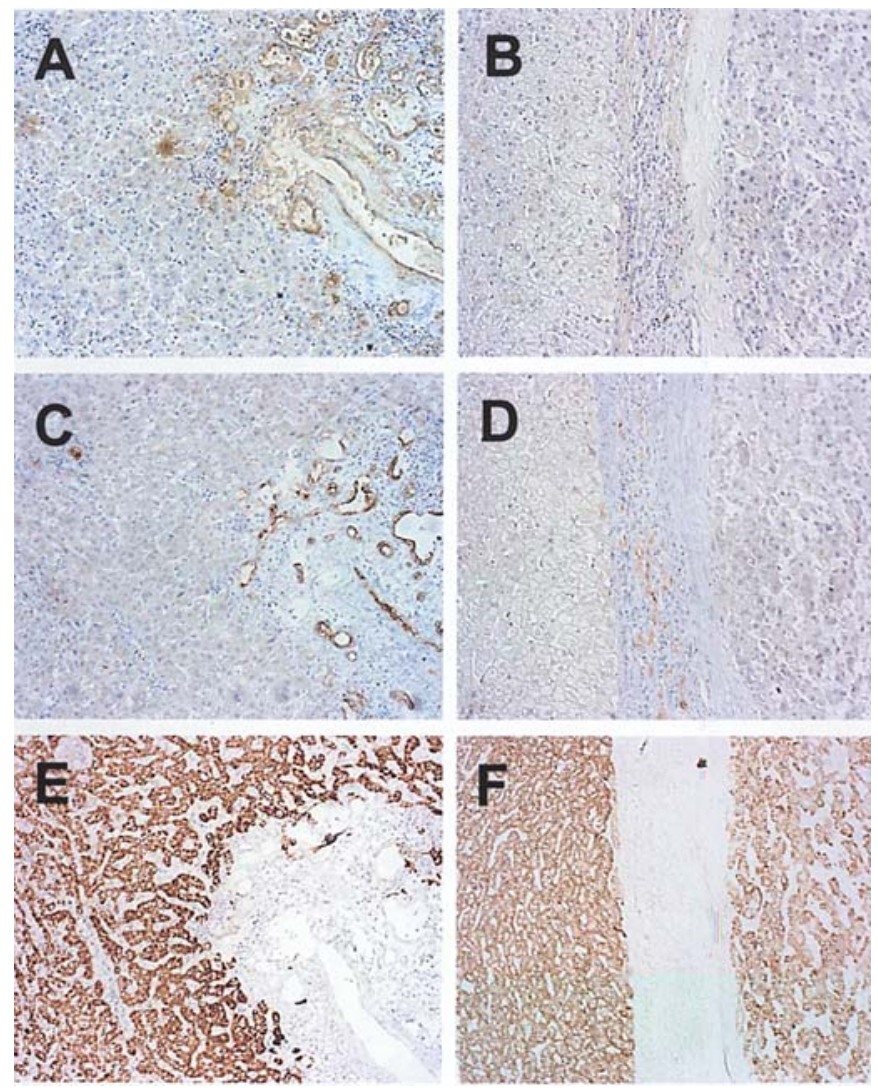

Figure 1. Immunostaining for KL-6 mucin (A and B), CK-7 (C and D), and Hep1 (E and F) in CC tissues (A, C, and E) and HCC tissues (B, D, and F). The carcinoma and the surrounding normal hepatic tissue are shown on the right and left side of each panel, respectively. Original magnification: x100.

(right side of Fig. 1C and D, respectively), as well as in the surrounding normal hepatic parenchyma (left side of Fig. 1C and D). In contrast, staining for Hep1 was observed in the normal hepatic parenchyma (left side of Fig. 1E and F) and in some HCC tissues (right side of Fig. 1F), but Hep1 was not positive in CC tissues (right side of Fig. 1E). The number of tumors showing positive staining is summarized in Table I.

Immunostaining of cHCC-CC for KL-6 mucin, $C K 7$, and Hep1. Typical immunostaining patterns of $\mathrm{cHCC}-\mathrm{CC}$ for KL-6, CK7, and Hep1 are shown in Fig. 3 and the number of tumors displaying positive staining of the cholangiocellular and hepatocellular areas is summarized in Table II. As can be seen in Fig. 3A, staining for KL-6 mucin was positive in the cholangiocellular tissue but not in the hepatocellular tissue. A similar staining profile was obtained in all of the cHCC-CC lesions examined (Table II). Conversely, staining for Hep1 was not found in any of the cholangiocellular tissues examined, but was seen in some hepatocellular tissues and all specimens of normal hepatic parenchyma (Fig. 3C). In contrast, as shown in Fig. 3B, CK7 was positive in both the cholangiocellular and hepatocellular tissues of cHCC-CC lesions.

\section{Discussion}

KL-6 mucin has been detected in various carcinomas and in normal secretory epithelial cells (5-8). Our previous studies

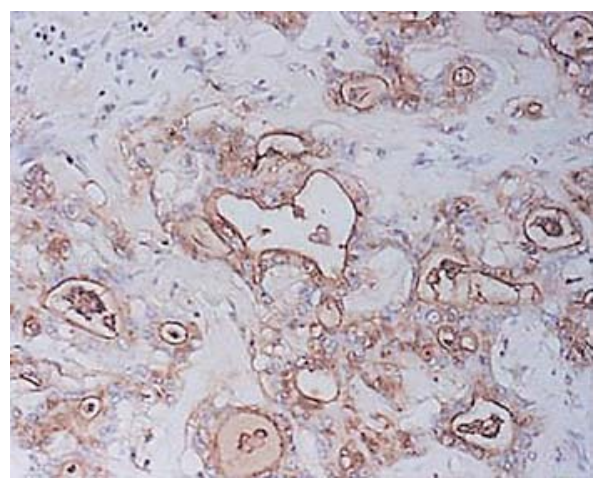

Figure 2. Immunostaining of CC tissues for KL-6 mucin. Original magnification: $\mathrm{x} 200$.
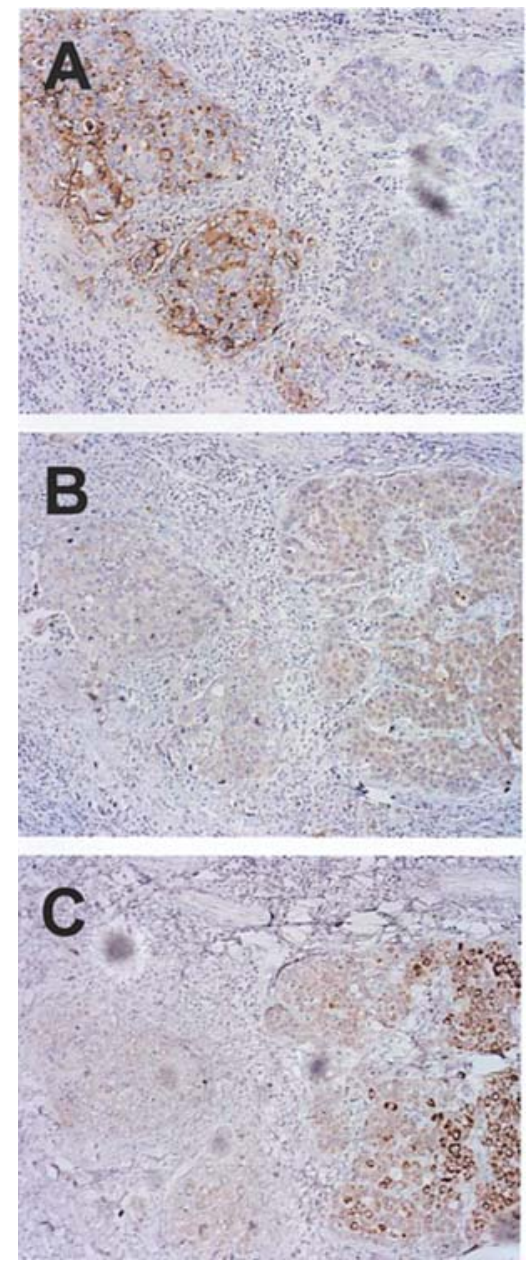

Figure 3. Immunostaining for KL-6 mucin (A), CK-7 (B), and Hep1 (C) in combined cHCC-CC tissues. The cholangiocellular and hepatocellular components are on the left and right side of each panel, respectively. Original magnification: x100.

showed that KL-6 mucin is expressed by primary colorectal carcinoma and ampullary carcinoma, and that aberrant expression of KL-6 mucin is associated with unfavorable clinicopathological characteristics, such as tumor invasion and metastasis as well as a worse prognosis (8). In the present study, we found that KL- 6 staining was positive in all of the 
CC tissues examined, while it was not positive in any of the HCC tissues examined (Fig. 1 and Table I). A similar selective pattern of KL-6 staining was also found in cHCC-CC tissues, and the cholangiocellular areas could be clearly detected by using KL-6 (Fig. 3 and Table II).

Primary liver cancer can be classified as HCC or intrahepatic CC. Since HCC and CC have different etiologic, epidemiologic, and clinical characteristics $(1,3)$, distinguishing between these two types of cancer is important. In combined cHCC-CC lesions with hepatocellular and cholangiocellular components, the distribution of these two tissues should be determined for classification of the tumor and assessment of the clinicopathological characteristics.

Several immunohistochemical studies have already been performed with $\mathrm{HCC}, \mathrm{CC}$, and $\mathrm{CHCC}-\mathrm{CC}$ to define the pathological characteristics of these tumors $(3,11-13)$. Hep1 is an antigen that exists in both normal hepatocytes and HCC tissues (14). The present study showed that $79.5 \%$ of HCC specimens and $25.0 \%$ of cHCC-CC specimens were positive for Hep1 expression in the hepatocellular tissues, while none of the $\mathrm{CC}$ or cHCC-CC specimens had positive cholangiocellular tissues (Table I and II). Similar selectivity for HCC and the hepatocellular tissues of cHCC-CC has also been reported for $\alpha$-fetoprotein (3).

The structural protein CK7 (part of the cytoskeleton) is often used for phenotyping of tumors together with CK20 (15). In the present study, staining for CK7 was observed in $95.2 \%$ of CC specimens and $35.9 \%$ of HCC specimens (Table I), although it was faint in some of the HCC specimens. Also, 58.3 and $25.0 \%$ of the cHCC-CC specimens were positive for CK7 in the cholangiocellular and hepatocellular tissues, respectively (Table II). This expression profile of CK7 was different from that previously reported showing predominant expression in CC and little expression in HCC $(16,17)$. This discrepancy may be due to the use of different criteria for selection of tumors with positive antigen expression, as pointed out elsewhere (17). Other immunoreactive substances, such as carcinoembryonic antigen, $\alpha 1$ antitrypsin, and fibrinogen, were reported to be detected at a high rate in both HCC and CC tissues (3). Therefore, these antigens and $\mathrm{CK} 7$ are inappropriate for distinguishing between $\mathrm{CC}$ and HCC.

KL-6 mucin is a type of MUC1, and it appears to be a good marker for separating $\mathrm{CC}$ from $\mathrm{HCC}$, since it is detected in $\mathrm{CC}$ tissues, but not in HCC tissues or normal hepatic parenchyma (Fig. 1A and B, and Table I). A similar distribution was also observed in combined cHCC-CC lesions, which showed positive staining in the cholangiocellular tissues but not in the hepatocellular tissues (Fig. 3A and Table II). Regarding MUC1 expression in CC tissues, a few investigations performed with mAbs for the core protein of MUC1 have suggested that increasing expression of MUC1 is related to unfavorable histopathological features and a poor prognosis $(18-21)$. In contrast, the KL-6 mAb used in this study recognizes an oligosaccharide moiety of MUC1 as a part of antigen and sialylation of this oligosaccharide is essential for recognition (6). KL-6 mucin was detected in all of the CC specimens examined in this study, while detection of MUC1 core protein was limited. It is likely that the sialylated oligosaccharides of MUC1 may be exposed on surface of the molecule, while the core protein is buried in the oligosaccharide moiety. Therefore, the higher sensitivity of KL-6 may be due to the position of its antigens in the MUC1 molecule.

In conclusion, staining of KL-6 mucin was observed in all CC specimens examined, but not in HCC specimens, suggesting that KL-6 may be a tumor marker for distinguishing CC from HCC. In addition, KL-6 was positive in the cholangiocellular tissues but not in the hepatocellular tissues of cHCC-CC; therefore, this antibody may be useful to detect the cholangiocellular component of $\mathrm{cHCC}-\mathrm{CC}$ and provide pathological information for selecting the clinical strategy.

\section{Acknowledgements}

This study was supported by Grants-in-Aid from the Ministry of Education, Science, Sports and Culture of Japan. We are also grateful to Atsuko Takeuchi for her excellent technical assistance.

\section{References}

1. The Liver Cancer Study Group of Japan: Primary liver cancer in Japan. Clinicopathologic features and results of surgical treatment. Ann Surg 211: 277-287, 1990.

2. Allen RA and Lisa JR: Combined liver cell and bile duct carcinoma. Am J Pathol 25: 647-655, 1949.

3. Goodman ZD, Ishak KG, Langloss JM, Sesterhenn IA and Rabin L: Combined hepatocellular-cholangiocarcinoma. A histologic and immunohistochemical study. Cancer 55: 124-135, 1985.

4. Jarnagin WR, Weber S, Tickoo SK, Koea JB, Obiekwe S, Fong Y, DeMatteo RP, Blumgart LH and Klimstra D: Combined hepatocellular and cholangiocarcinoma: demographic, clinical, and prognostic factors. Cancer 94: 2040-2046, 2002.

5. Kohno N: Serum marker KL-6/MUC1 for the diagnosis and management of interstitial pneumonitis. J Med Invest 46: 151-158, 1999.

6. Kohno N, Akiyama M, Kyoizumi S, Hakoda M, Kobuke K and Yamakido M: Detection of soluble tumor-associated antigens in sera and effusions using novel monoclonal antibodies, KL-3 and KL-6, against lung adenocarcinoma. Jpn J Clin Oncol 18: 203-216, 1988 .

7. Tang W, Inagaki Y, Kokudo N, Guo Q, Seyama Y, Nakata M, Imamura H, Sano K, Sugawara Y and Makuuchi M: Aberrant expression of KL-6 in carcinoma of the ampulla of Vater: association with cancer progression. World J Gastroenterol 11: 5450-5454, 2005.

8. Guo Q, Tang W, Inagaki Y, Midorikawa Y, Kokudo N, Sugawara Y, Nakata M, Konishi T, Nagawa H and Makuuchi M: Clinical significance of subcellular localization of KL-6 mucin in primary colorectal adenocarcinoma and metastatic tissues. World J Gastroenterol 12: 54-59, 2006.

9. Liver Cancer Study Group of Japan: The General Rules for the Clinical and Pathological Study of Primary Liver Cancer. 2nd edition. Kanehara, Tokyo, 2003.

10. Sobin LH and Wittekind C: TNM Classification of Malignant Tumors. 5th edition. Wiley-Liss, New York, 1997.

11. Maeda T, Adachi E, Kajiyama K, Sugimachi K and Tsuneyoshi M: Combined hepatocellular and cholangiocarcinoma: proposed criteria according to cytokeratin expression and analysis of clinicopathological features. Human Pathol 26: 956-964, 1995.

12. Shibahara H, Tamada S, Higashi M, Goto M, Batra SK, Hollingsworth MA, Imai K and Yonezawa S: MUC4 is a novel prognostic factor of intrahepatic cholangiocarcinoma-mass forming type. Hepatology 39: 220-229, 2004.

13. Wee A: Fine needle aspiration biopsy of the liver: algorithmic approach and current issues in the diagnosis of hepatocellular carcinoma. Cyto J 2: 7, 2005.

14. Vessey CJ and de la Hall PM: Hepatic stem cells: a review. Pathology 33: 130-141, 2001. 
15. Moll R, Franke WW, Schiller DL, Geiger B and Krepler R: The catalog of human cytokeratins: patterns of expression in normal epithelia, tumors and cultured cells. Cell 31: 11-24, 1982.

16. Maeda T, Kajiyama K, Adachi E, Takenaka K, Sugimachi K and Tsuneyoshi M: The expression of cytokeratins 7, 19, and 20 in primary and metastatic carcinomas of the liver. Mod Pathol 9: 901-909, 1996.

17. Centeno BA: Pathology of liver metastasis. Cancer Control 13: 13-26, 2006.

18. Sasaki M, Nakanuma Y and Kim YS: Characterization of apomucin expression in intrahepatic cholangiocarcinomas and their precursor lesions: an immunohistochemical study. Hepatology 24: 1074-1078, 1996.
19. Higashi M, Yonezawa S, Ho JJL, Tanaka S, Irimura T, Kim YS and Sato E: Expression of MUC1 and MUC2 mucin antigens in intrahepatic bile duct tumors: its relationship with a new morphological classification of cholangiocarcinoma. Hepatology 30: 1347-1355, 1999.

20. Matsumura N, Yamamoto M, Aruga A, Takasaki K and Nakano M: Correlation between expression of MUC1 core protein and outcome after surgery in mass-forming intrahepatic cholangiocarcinoma. Cancer 94: 1770-1776, 2002.

21. Yuan SF, Li KZ, Wang L, Dou KF, Yan Z, Han W and Zhang YQ: Expression of MUC1 and its significance in hepatocellular and cholangiocarcinoma tissue. World J Gastroenterol 11: 4661-4666, 2005. 